\title{
Framework of Designing an Adaptive and Multi- Regime Prognostics and Health Management for Wind Turbine Reliability and Efficiency Improvement
}

\author{
B.L.Song, J.Lee \\ National Science Foundation (NSF) Center for Intelligent Maintenance System (IMS) \\ Cincinnati, US
}

\begin{abstract}
Wind turbine systems are increasing in technical complexity, and tasked with operating and degrading in highly dynamic and unpredictable conditions. Sustaining the reliability of such systems is a complex and difficult task. In spite of extensive efforts, current prognostics and health management (PHM) methodologies face many challenges, due to the complexity of the degradation process and the dynamic operating conditions of a wind turbine. This research proposed a novel adaptive and multi-regime prognostics and health management (PHM) approach with the aim to tackle the challenges of traditional methods. With this approach, a scientific and systematic solution is provided for health assessment, diagnosis and prognosis of critical components of wind turbines under varying environmental, operational and aging processes. The system is also capable of adaptively selecting the tools suitable for a component under a certain health status and a specific operating condition. The adopted relevant health assessment, diagnosis and prognosis tools and techniques for wind turbines are warranted by the intensive research of PHM models by the IMS center for common rotary machinery components. Some sub-procedures, such as information reconstruction, regime clustering approach and the prognostics of rotating elements, were validated by the best score performance in PHM Data Challenge 2008 (student group) and 2009 (professional group). The success of the proposed wind turbine PHM system would greatly benefit current wind turbine industry.
\end{abstract}

Keywords-PHM; Adaptive tool selection; Multi-regime prognostics; Information reconstruction; Holo-coefficient

\section{INTRODUCTION}

Wind energy is an unlimited, renewable and clean energy source and makes it possible to establish a large number of Megawatts in a relatively short time. Wind energy has become a progressively more competitive source of energy. The American wind energy association reported that wind percentage in all the new capacity added increased from $2 \%$ in 2004 to $42 \%$ in 2008 (Figure 1). It is remarkable that the United States and China have now become the leaders in the wind power market industry in terms of newly installed capacity, surpassing Germany, the previous leader in wind power (Figure 2). The US market's new wind energy converter installations, reaching up to $8.5 \mathrm{GW}$ at the end of 2008, have increased the total wind power generating capacity by half when compared to the previous year. Such a notable feat is due in part to the US Department of Energy's 2008 report [1] purporting that the power that can be harvested from the country's wind resources has the potential to supply $20 \%$ of its domestic demand for electricity.

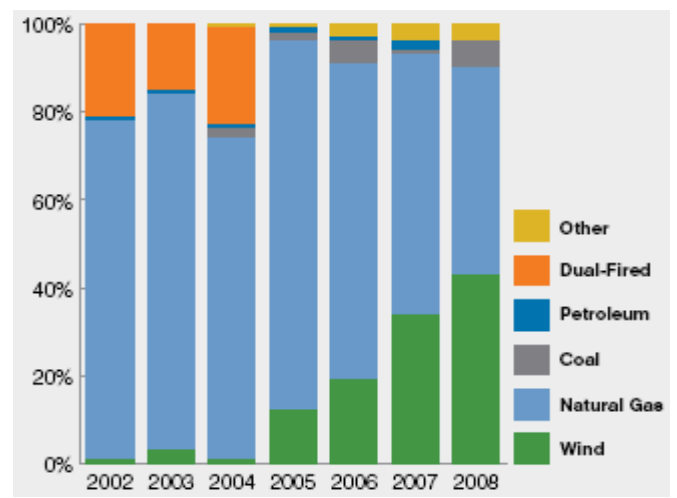

Fig. 1. Percentage Of New Capacity Additions (Source: AWEA-Annual Wind Industry Report 2009)[2]

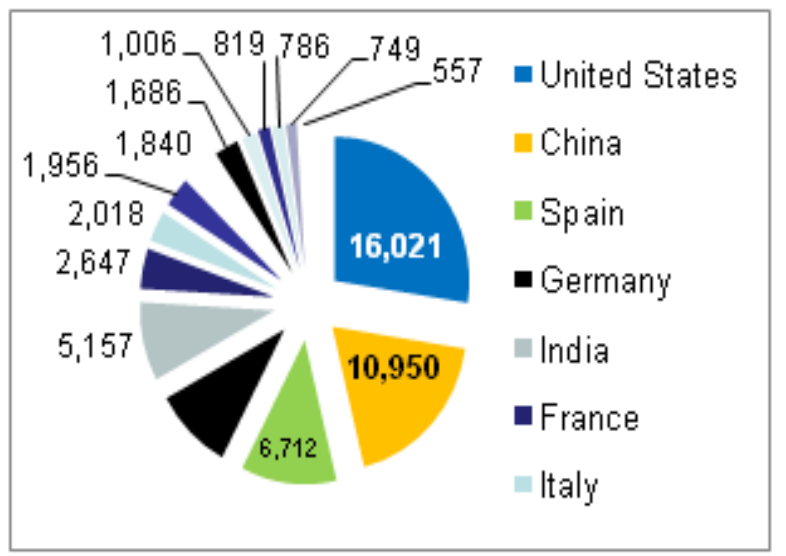

Fig. 2. Total increased wind turbine capacity from 2005 to 2008

The increased reliance on wind energy as an energy source for the world makes the increased uptime and reliability of wind turbine systems become more critical issues. The degradation of wind turbine critical components' health in the dynamic operating conditions could badly impact the wind energy generation efficiency. In recent years, increasing 
interests have been put on researching the condition monitoring and health management technologies based on the fact that the preventing the failure of critical equipments in advance could result in a significant amount of time and cost savings, and the overall improved reliability and safety of operations.

Ciang et al. provided a review of fault detection approaches for wind turbines, such as, acoustic emission events method, thermal imaging method, ultrasonic methods, various modal-based approaches, fiber optics method, laser Doppler vibrometer method, electrical-resistance based damage detection method, strain memory alloy method, $x-$ radioscopy method and eddy current method [3]. The techniques surveyed focus more on physical models for structural health monitoring. In recent decade, with the spread of artificial intelligent and machine learning technologies, data driven methods, which base on the analysis of signals (e.g. vibration from accelerometers) to assess the asset's health degradation status, diagnose current failure modes, and predict future health, have gained wide attention for their success in rotary machines [4][5][6].

In spite of extensive efforts by current prognostics and health management (PHM) methodologies, based on both data-driven and physical models, a successful deployment of these existing techniques to wind turbine applications still faces many challenges, such as, the complexity of the wind turbine health degradation and the dynamic operating conditions of a wind turbine. Wind turbine systems are increasing in technical complexity, and are tasked with operating in highly dynamic and unpredictable operating conditions.

Each component may degrade to various health statuses even under a same operating condition. Multi-regime approach is necessary to be researched to extend traditional health management techniques to consider the dynamic behavior of these components by segmenting the component health aging and operating conditions to various operating regimes. Moreover, considering the health management models may not have the same performance on different regimes, the selection of the proper model for a certain component under a specific application condition is significant. In practice, health representative data collected is a complicated and energy wide-range distributing signal. Only some parts of the signal related to the particular regime are of interest. In order to remove or reduce noise, a novel and effective information reconstruction method is desired to filter and reassemble the signal components without losing the information of interest.

An investigation of current industrial health management systems was conducted for the largest wind turbine manufacturers, namely GE Energy, Vestas and Siemens [7][8][9]. It is found that GE Energy's system provides more advanced functionalities in diagnosis and fault detection for drive train components than the others. Nevertheless, some functions, such as, system degradation assessment and failure prediction based predictive condition monitoring, adaptive and multi-regime prognostics for dynamic conditions, are nonexistent.

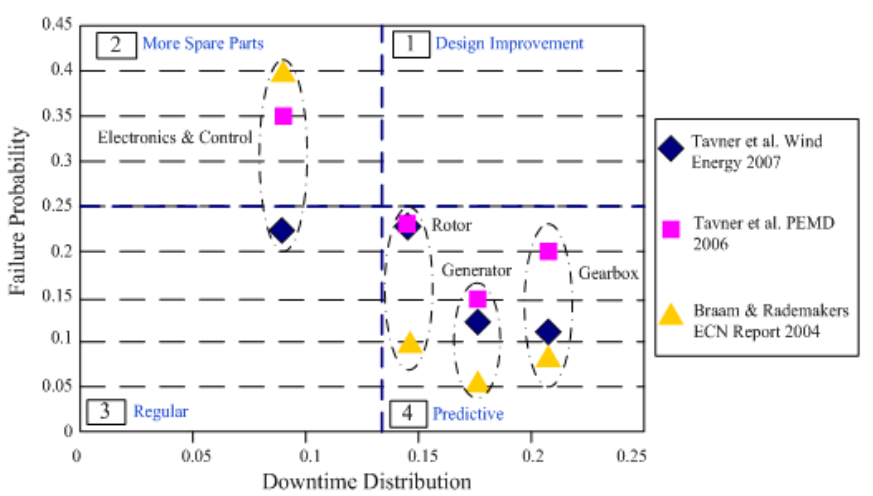

Fig. 3. Survey of critical components of wind turbines[10][11][12]

Numerous surveys conducted on faults experienced by wind turbine components have revealed that gearbox, generator and rotor blades have longer downtime once they fail. (Figure 3). Maintenance costs are estimated to make up approximately $15 \%$ of production costs in many industries. In the survey of the failure modes and hot spots of the critical component gearbox conducted by Musial [13], almost 100\% of the 500 to $900 \mathrm{~kW}$ gearbox designs have had at least one retrofit/design change to the high speed bearing arrangement. More than half change happened on planet bearings and intermediate shaft locating bearings. A more accurate health estimation of critical components of wind turbines would be significant for their reliability improvement and breakdown cost reduction.

In order to minimize breakdown performance and associated maintenance and logistics costs, and improve efficiency of power generation and safety considerations, an adaptive and multi-regime prognostics and health management (AMPHM) approach is proposed in this paper. The proposed wind turbine AMPHM is a data driven method for health assessment, diagnosis and prognosis through the analysis of vibration signals from accelerometers. Its key technology was validated upon the research that resulted in the first place award in the international PHM Challenge in 2009. The proposed AMPHM system aims to provide an effective and systematic solution to improve the uptime and reliability of wind turbines working under the varying environmental, operational and aging processes, which will make a breakthrough for traditional PHMs.

This paper is structured as follows: the architecture of the adaptive and multi-regime wind turbine PHM system, which consists of data acquisition, health management, and health visualization, is firstly presented in section 2 . The data acquisition is introduced in section 2 too. Health management details, including adaptive tool selection and multi-regime PHM, are explained in sections 3 and 4 respectively. Section 5 takes gearbox as an example to demonstrate the health visualization. A conclusion is made at the end section

\section{ARChitecture Of AdAPtive AND Multi-Regime WIND TURBINE PHM SYSTEM}

The architecture of adaptive and multi-regime wind turbine prognostics and health management system working in remote and online mode is proposed in Figure 4. Inside Nacelle, the 
up-tower data acquisition sub-system continuously collects signals, including both the operating conditions and the system behaviors, from installed sensors, as well as the wind turbine control system. Data files are transmitted through wind farm network from the nacelle to the remote health management database and server, where, the data files are downloaded and processed for health assessment, diagnosis and prognosis. The processing result will be visualized in a user-friendly humanmachine interface (HMI) on client computer so that users can immediately understand and determine the health condition the critical components, and decision aid actions (e.g. predictive maintenance) can be taken on wind turbines timely, and accordingly.

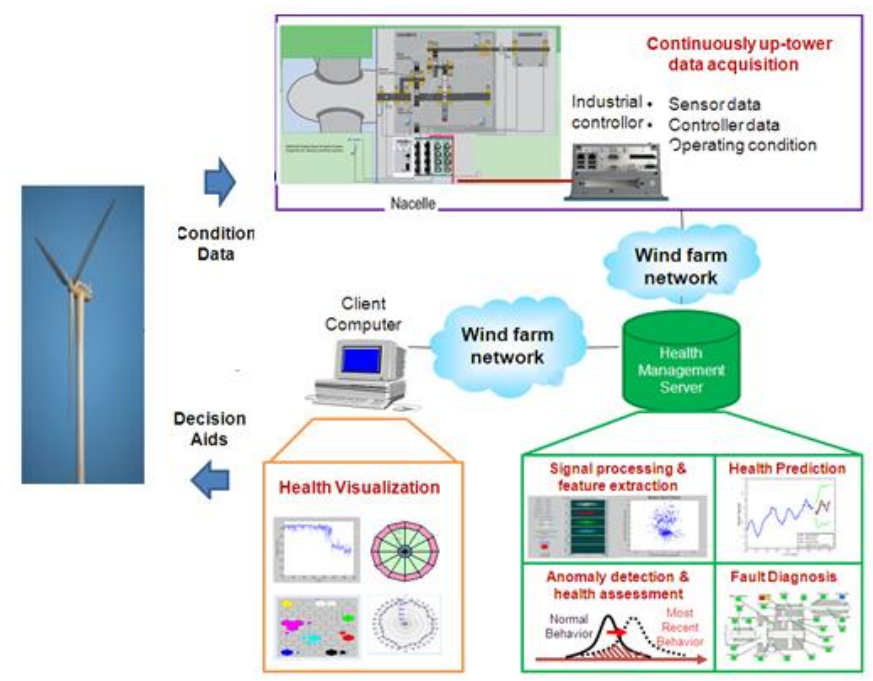

Fig. 4. Architecture of adaptive and multi-regime wind turbine PHM System

The proposed instrumentation plan for the up-tower condition monitoring sensor data acquisition (DAQ) for the critical components of wind turbine, gearbox and generator, is demonstrated in Figure 5. One low frequency PCB IEPE accelerometer, typically with sensitivity $500 \mathrm{mV} / \mathrm{g}$ and frequency span $0.2-3000 \mathrm{~Hz}$, is placed on the main shaft (blade passing, main bearing) to collect vibration signals. Five PCB IEPE accelerometers with sensitivity $100 \mathrm{mv} / \mathrm{g}$ and frequency span of $0.5-15000 \mathrm{~Hz}$ are positions on the gearbox and generator which have higher rotational speeds and gear mesh frequencies. One PCB laser tachometer is used to monitor the main bearing speed and synchronize the vibration. Three Compact DAQ NI USB 9234 (4 channels) are adapted for accelerometers and the tachometer signal collection. An industrial controller (NI 3110) is sitting in the Nacelle connected to NI USB 9234 to collect data continuously.

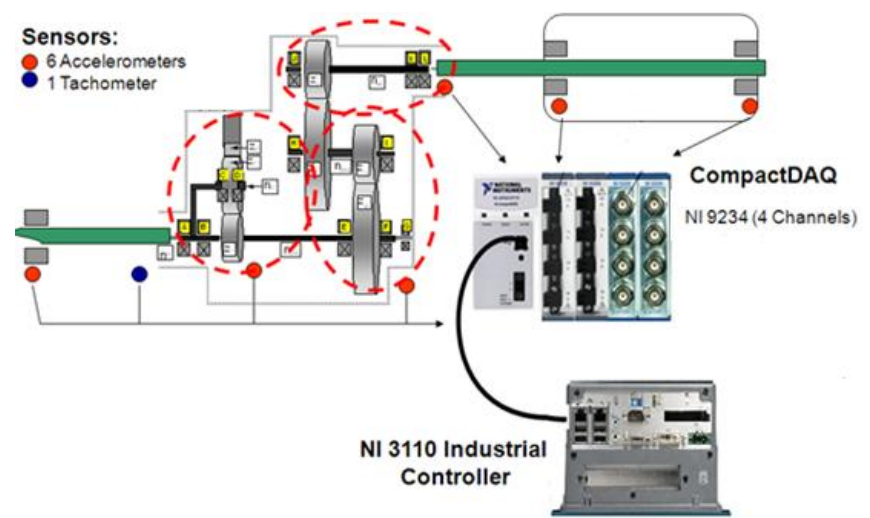

Fig. 5. Data acquisition solution (source: $N I$ )

\section{TECHNICAL APPROACH}

Figure 6 illustrates the flow of the proposed wind turbine PHM which is conducted on health management server. As rotational components of a wind turbine work under dynamic conditions, an adaptive tool selection agent is designed to select the proper tool for a certain component under a specific situation. With the multiple operating regimes segmented, the health representative features are extracted and fed into the corresponding health assessment models. After health assessment, multi-dimensional features will be converted into a 1-Dimention health index between 0 and 1, with 1 indicating a perfect health condition and 0 indicating an unacceptable heath condition. The history of the health index can then be further processed for fault diagnosis and health prediction.

An illustrative example is presented in the following sections to demonstrate the procedure of the adaptive tool selection, multi-regime PHM approach, and the health visualization at users' end as well.

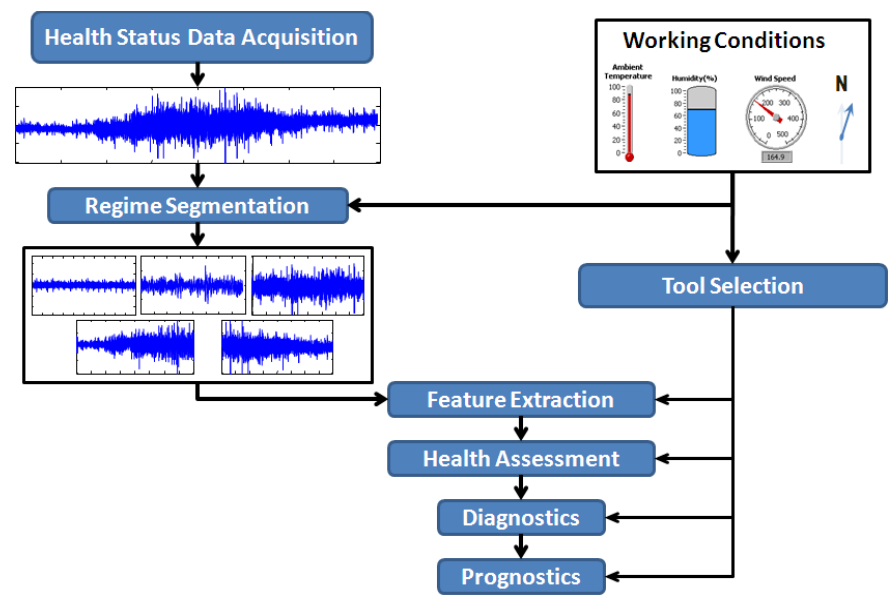

Fig. 6. Flow of adaptive and multi-regime wind turbine PHM 


\section{A. Adaptive Model Selection}

Due to constantly changing operating conditions, such as wind speed and wind direction, as well as slow evolving environmental conditions such as temperature, the electrical and mechanical system of a wind turbine actually operates under highly dynamic conditions (Figure 7a). These operating conditions will be classified into different regimes in the following study. On each operating regime, wind turbine will experience different health statuses during degradation (Figure $7 b$ ). The combinations of various regimes and corresponding health statuses constitute the multiple application conditions. Considering a wind turbine has multiple components, and the performance of different PHM models may be sensitive to application conditions, the selection of the proper model for a certain component under a specific application condition is significant. An adaptive tool selection agent (Figure 7c) is designed for this purpose. This agent self adapts to the input of the customer requirements (e.g. implementation cost, human involvement, system knowledge, computation efficiency and signal sampling frequency, etc.) and application conditions, and automatically identifies a particular model that is suitable for that specific condition.

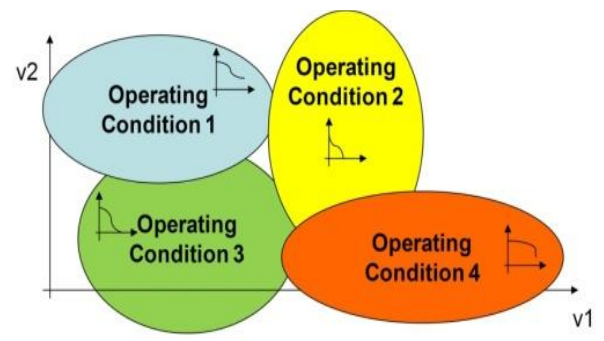

a) Multiple operating regimes

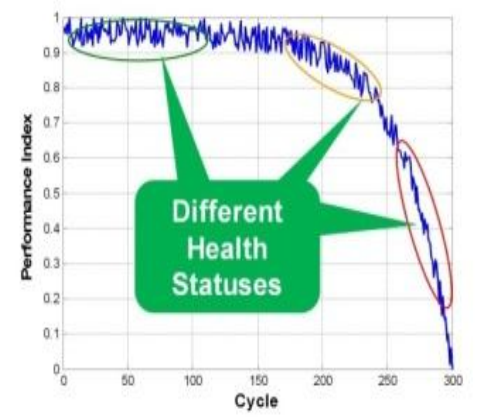

b) Multiple health degradation statuses under a certain operating regime

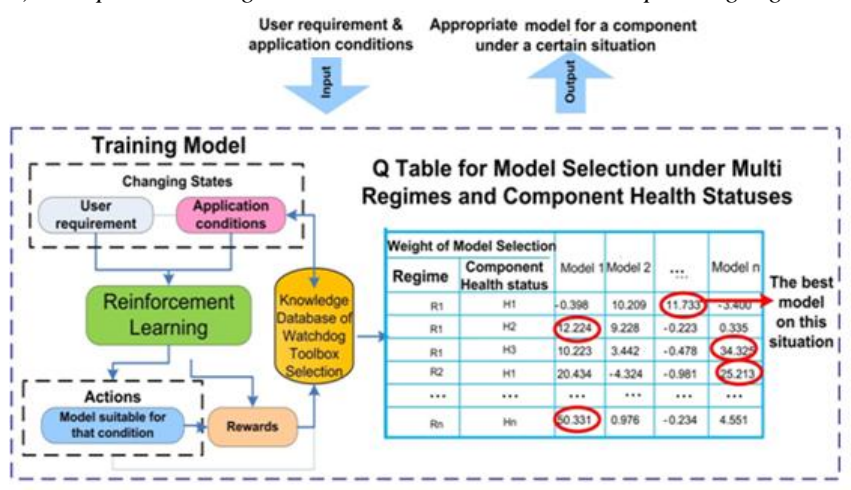

c) Adaptive Tool Selection

Fig. 7. Operating regime and analyze tool
The expert knowledge of various situations that a particular model is suitable to use will be structured to initialize a model selection rules knowledge base.

Taking the customer requirements and applications conditions as inputs, and the models used in those situations as outputs, a reinforcement learning model will be trained to learn the model selection knowledge under different situations [14].

Formally, the basic reinforcement learning model according to Wikipedia consists of:

1) a set of environment states $S$ (i.e. a set of user requirements and application conditions);

2) a set of actions $A$ (i.e. a set of models suitable for that state); and

3) a set of scalar "rewards" in $\mathbb{R}$.

The environment is typically formulated as a finite-state Markov Decision Process (MDP). At each time $t$, the reinforcement learning agent perceives its state $s_{t} \in S_{\text {and }}$ the set of possible actions $\boldsymbol{A}\left(\boldsymbol{s}_{t}\right)$. It chooses an action $a \in A\left(s_{t}\right)$ and receives from the environment the new state $\boldsymbol{s}_{\boldsymbol{t}+1}$ and a reward $\boldsymbol{r}_{\boldsymbol{t}+1}$.

Based on these interactions, the reinforcement learning agent must develop a policy $\pi: S \rightarrow$ Awhich maximizes the quantity $R=r_{0}+r_{1}+\cdots+r_{n}$ for MDPs which have a terminal state, or the quantity.

The adaptive tool selection agent selects the most appropriate model for each application condition by choosing the largest Q-value for all the application condition /model pairs in the row of that application condition. The Q-value is determined by the sum of the (maybe discounted) reinforcements received when performing an action following a given policy.

Model selection rules will then be updated and structured into a knowledge base again. The reinforcement learning agent will iterate when receiving a new state.

\section{B. Multi-regime Prognostics and Health Management}

The changing operating conditions have significant influence on the baseline of a data-driven wind turbine PHM model. The relationship between the operating conditions and the model baseline is very hard to be established analytically or experimentally. To conquer the problem, it is proposed to use the operating regime approach [15] to employ multiple simple PHM models developed for static operating conditions to deal with dynamic operating conditions, which will be referred to as multi-regime PHM here.

The procedure of multi-regime prognostics is illustrated in Figure 8. First, the collected data, including those indicating system operating conditions and those indicating system behaviors, will be used to identify what regime the system is operating in. If the new measurement cannot fit to any of the existing patterns, a new operating regime will be learned. Once the operating regime is identified, the data will be fed into the corresponding PHM model that has either been established, or will have to be created for a newly-learned 
operating regime. The PHM models include sub-procedures, such as feature extraction, health assessment and fault diagnostics, which have been studied and applied intensively by the IMS center for common rotary machinery components. Finally, the health indices obtained from different operating regimes will be fused together to form a continuous time series of the system health, which will be used for health prediction.

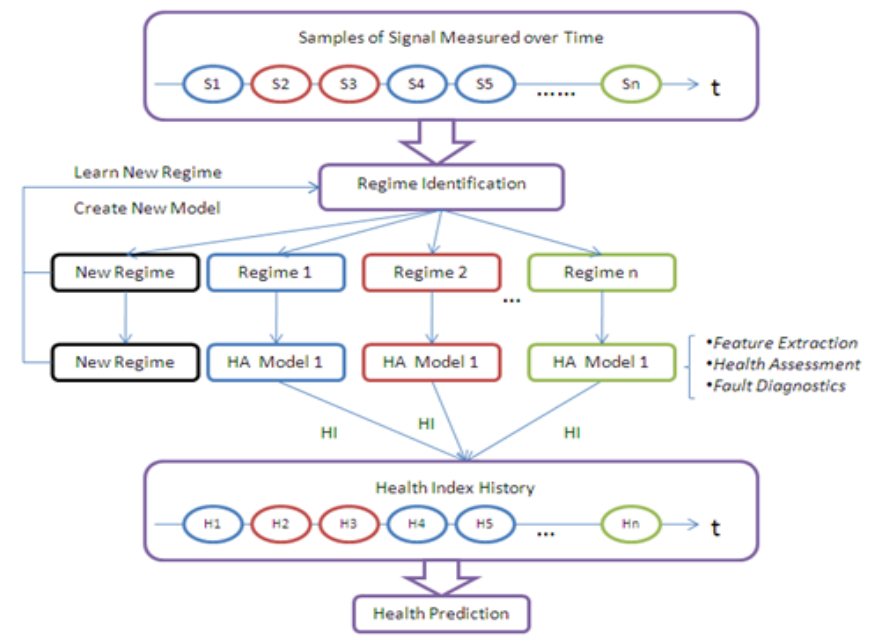

Fig. 8. Multi-regime Wind Turbines Health Assessment and Prediction

\section{1) Signal Processing}

Due to frequent environmental changes and unpredictable wind turbulence, as well as the frequent stopping and starting of a wind turbine, recorded raw sensor data are not appropriate as direct input to various signal processing tools, even though they are continuous vibration or acoustic time series. In order to enhance data quality and improve signal processing efficiency, data preprocessing, such as de-noising and data segmentation, is a necessary preliminary step for signal processing. In order to remove or reduce noise and effects from other unrelated sources, de-noising can be performed by using noise smoothing filters or band pass filters, based on knowledge of the frequency distribution of wind turbine signals. Data segmentation can be performed by using working condition data to sift and retain the active vibration or acoustic time series.

To detect changes in the vibration signatures caused by abnormal behaviors, recorded data can be analyzed in the time domain. A number of simple signal metrics based on the time domain waveform, such as peak level, root mean square (RMS) value and kurtosis, have widespread applications in condition monitoring and fault detection for wind turbines. However, in order to gain more comprehensive knowledge and extract more reliable and effective health indicators for wind turbines, signals need to be observed in the frequency domain and time-frequency domain. Fast Fourier Transform (FFT) and cepstral analysis are applied for frequency domain analysis to decompose a signal into its component frequencies and amplitudes, and to isolate individual components of a complex signal for easier pattern identification. Spectral analysis of the electric power and of accelerations measured in the wind turbine system is performed effectively for stationary signal analysis where energy variation over time is dispensable. For non-stationary, non-linear time series which can be observed from time to time in wind turbine systems under varying environmental and operating conditions, more advanced signal processing tools in time-frequency domain such as short-time Fourier Transform (STFT), Wigner-Ville distribution (WVD), Wavelet Transform (WT), and HilbertHuang transform (HHT) are applied. A time-frequency representation is a view of a signal represented over both time and frequency. In a wind turbine system, non-stationary, nonliner time series often appear during the transient period between different working conditions, and they usually carry abundant dynamic information of the system. The aforementioned advanced signal processing tools, especially WT and HHT methods, are able to capture the transient characteristics of non-stationery vibration data and are suitable for impact detection caused by a stroke of lightning, a collision with a large bird, or wave-induced tower oscillations of off-shore plants.

\section{2) Feature Selection}

After signal processing, various features can be extracted such as mean, peak, RMS in time domain, characteristic frequency, amplitude and phase in frequency domain and energy-time-frequency distribution in time-frequency domain. There may be, however, a lack of quality features, or there may be redundant features which increase feature dimension and affect the efficiency of the prognostics and health management activity. Feature selection has become the focus for applications in which tens or hundreds of variables are available. Appropriate feature selection can improve the data mining performance, help data visualization and reduce dimensionality and noise. For wind turbines, feature selection is further complicated by the fact that a wind turbine system is usually operating in varying environmental conditions, under diverse operating conditions and experiencing different aging processes. To establish an accurate and effective feature set to facilitate operation regime segmentation and health assessment of critical rotary components, it is necessary to identify operating condition parameters and select damage-sensitive features.

For feature selection, four methods are proposed; principal component analysis (PCA), fisher criterion, SVM-based feature selection and GA-based feature selection. PCA transforms a number of possibly correlated variables into a smaller number of uncorrelated variables, called principal components, and usually involves the calculation of the eigenvalue decomposition of a data covariance matrix, or the singular value decomposition of a data matrix after mean centering the data for each attribute. Fisher criterion seeks the features that are efficient for discrimination to minimize the within-class distance and maximize the between-class distance. The objective of the SVM-based feature selection method is to find a subset of size $r$ among d variables $(r<d)$, which maximizes the performance of the classifier and predictor. This method is a backward sequential selection approach. It starts with all the features and removes one feature at a time until only $r$ features are left, and until the performance of the classifier and predictor are maximized. In a GA-based approach, a given feature subset is represented as a binary string of length $d$, with a zero or one in position $i$ 
denoting the absence or presence of feature $\mathrm{i}$ in the set. A population of chromosomes is maintained. Each chromosome is evaluated to determine its "fitness". Based on a "fitness" threshold setting, an optimized binary string can be obtained to describe the feature selection result.

\section{3) Information Reconstruction}

The mechanical drive-train of a wind turbine is a very complex system that can generate vibrations from its various elements, such as gears, shafts, and bearings. Transmission path effect, signal coupling, and noise contamination can further induce difficulties in the development of a PHM system for wind turbines. In practice, vibration data collected by accelerometer is a complicated and energy wide-range distributing signal. But only some parts of the signal related to the particular machine condition are of interest. In order to remove or reduce noise and effects from other unrelated sources and further enhance signal components of interest, a novel information reconstruction method for filtering and reassembling the signal components to reconstruct signal without losing the information of interest is introduced. This method can also work for signal clustering and wind turbine diagnosis in varying operating conditions.

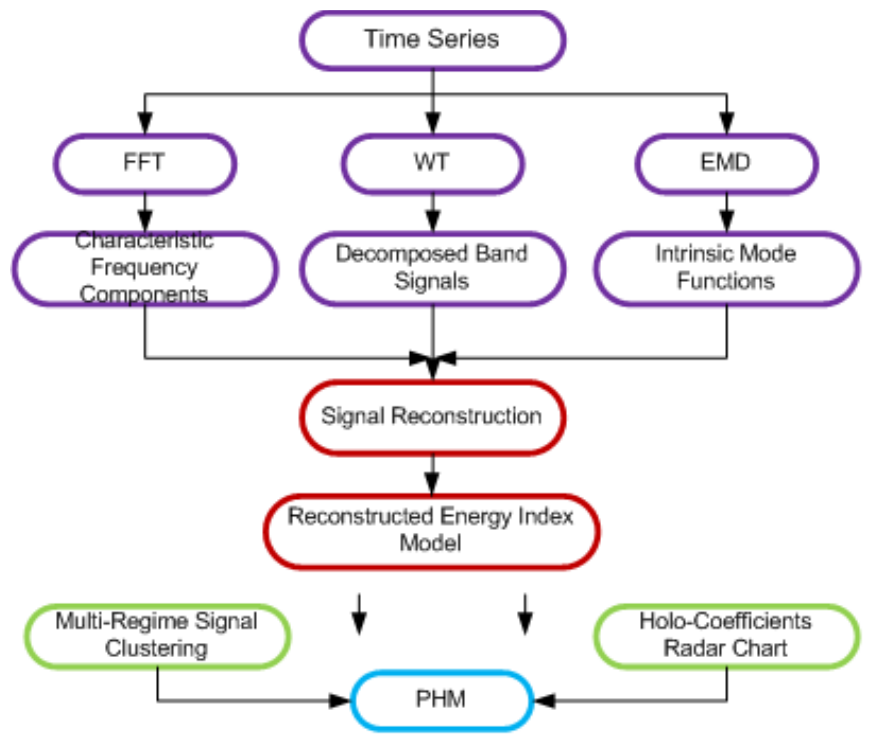

Fig. 9. Information Reconstruction

Firstly, the signal is transformed from time domain to frequency domain or time-frequency domain with FFT, WT, or HHT. Then, reconstruction filters are employed to sift the frequency components in FFT spectrum, to sift the decomposed band signals from WT analysis, or to sift the intrinsic mode functions (IMFs) from empirical mode decomposition (EMD) process of HHT. Next, sifted signal components are reassembled together to reconstruct a new signal.

\section{4) Regime Clustering}

Energy coefficients are then calculated for the reconstructed energy index model, in which energy coefficients are selected to have certain classification power. The basic idea is to identify and further classify the data with similar attributes to the same specified group. Moreover, energy coefficients are also supposed to be comprehensible for the user or have physical meaning. This is necessary whenever the classified pattern is to be used for supporting a decision to be made. Knowledge comprehensibility can be achieved by using high-level knowledge representations from experts or historic data resources.

Then, correlation analysis (CA) and distance measurement (DM) techniques are utilized to cluster signals under diverse shaft speeds and loads. CA on two energy coefficient vectors is defined as

$$
C A=\left(C_{E i} \cdot C_{E j}\right) /\left(\left|C_{E i}\right| *\left|C_{E j}\right|\right)
$$

where $\cdot$ means dot product, $|\bullet|$ means the largest singular value of a vector. The result of CA ranges between zero and one, with higher CA signifying a higher correlation.

DM on two signals is

$$
D M=\left\|C_{E i}-C_{E j}\right\|
$$

where $\|\bullet\|$ is the Euclidean distance, with lower DM signifying a higher similarity.

\section{5) Diagnosis}

Finally, energy coefficients are used as health indicators in holo-coefficients radar chart for the purpose of health assessment and prognostics of rotating elements in the wind turbine mechanical drivetrain. A holo-coefficients radar chart consists of all the energy coefficients. The multivariate coefficients are displayed in radar chart starting from the same point and in different equi-angular spokes, with each spoke representing one of the variables. The data length of a spoke is proportional to the magnitude of the variable. In the chart, the contribution rate of each coefficient can be revealed very clearly along with its variation with operating conditions. Figure 10 shows an example of using holo-coefficients radar chart to identify two patterns. In Pattern A, input shaft unbalance (radials 1 and 19) and bearing outer defect at input shaft output side (radial 21) are diagnosed. Figure 10 (b) shows the holo-coefficients radar chart of another pattern (Pattern B). It is determined that this pattern contains gear error defect at idler shaft 2 location (radials 8 and 26). 

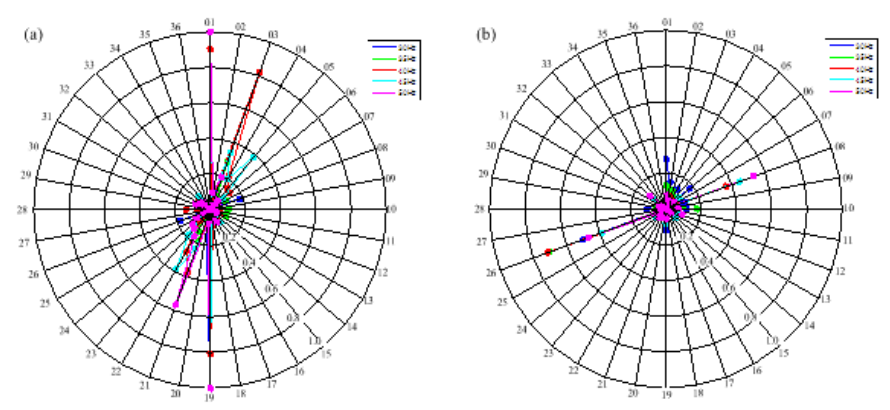

Fig. 10. Holo-coefficients Radar Chart of (a) Pattern A, (b) Pattern B

\section{6) Prognosis}

The similarity-based prognostics (SBP) approach is adopted from The Watchdog Agent ${ }^{\circledR}$ toolbox developed by the IMS Center for predicting future values of health indicator under dynamic operating conditions [16].

This similarity based prognostic technique was validated in the 2008 Prognostics and Health Management Society data challenge in which it produced the best prediction estimates and achieved number one overall in the competition [17]. By using multiple SBP models under different regimes, the given sensor data are fused into a single time series of health indices, which is then used in multi-regime SBP model.

\section{HEalth Visualization}

An illustrative example is taken to demonstrate the visualization of the detection of mechanical faults and prediction of future health for a generic gearbox using accelerometer data and information about bearing geometry. On the first step (Figure 11), the operating conditions are classified to different regimes, and the vibration signals collected from gearbox is segmented to corresponding regimes.

The second step in Figure 12 applies the adaptive tool selection approach and selects the time domain, frequency domain and wavelet analysis tools to process the raw data in different regimes and extract corresponding features. The third step, Figure 13, selects the principle features, reconstructs signals and generates energy coefficients, which are used as health indicators in holo-coefficients radar chart for the purpose of health assessment and diagnosis. A fault of chipped tooth problem in the first gear, for instance, is diagnosed. Also higher risk in high speed regime is alarmed. And the last step as in Figure 14, similarity based Match Matrix method is chosen to learn the health pattern based on the historical runs of similar gearboxes, and predict the future health.

The above mentioned information reconstruction and regime clustering approach, and the prognostics of rotating elements, were validated by the best score performance in the PHM Data Challenge Competitions 2008 (student group) and 2009 (professional group)[18].

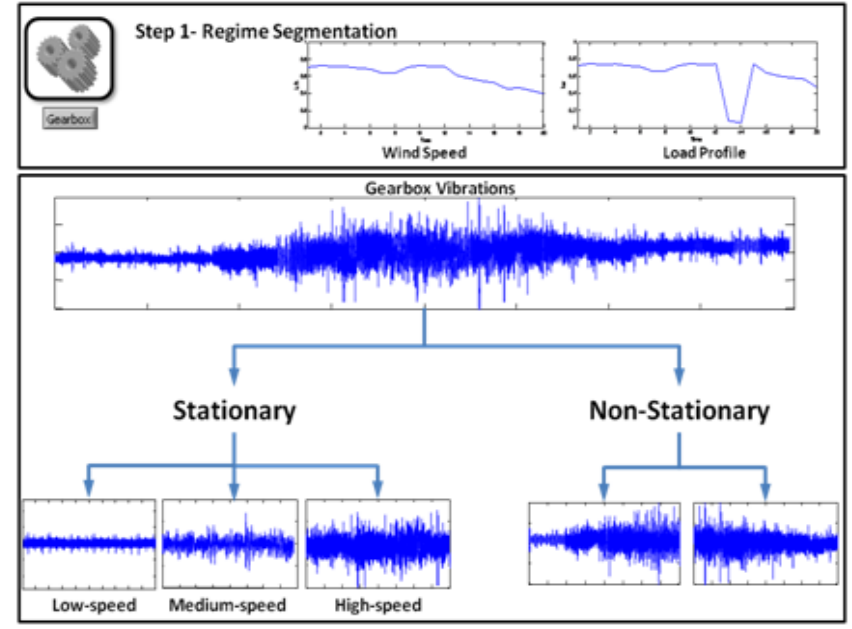

Fig. 11. Step 1 of wind turbine PHM: regime segmentation

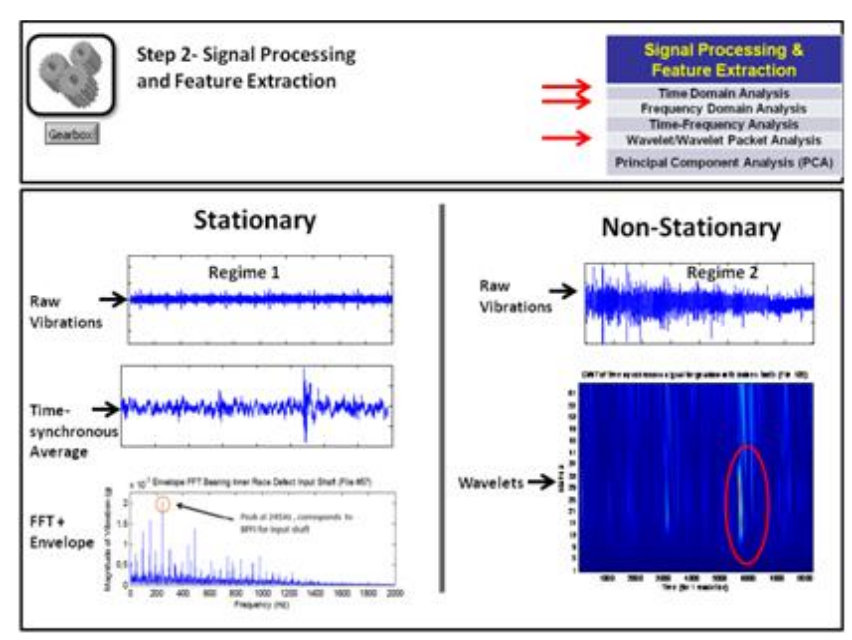

Fig. 12. Step 2 of wind turbine PHM: signal processing and feature extraction

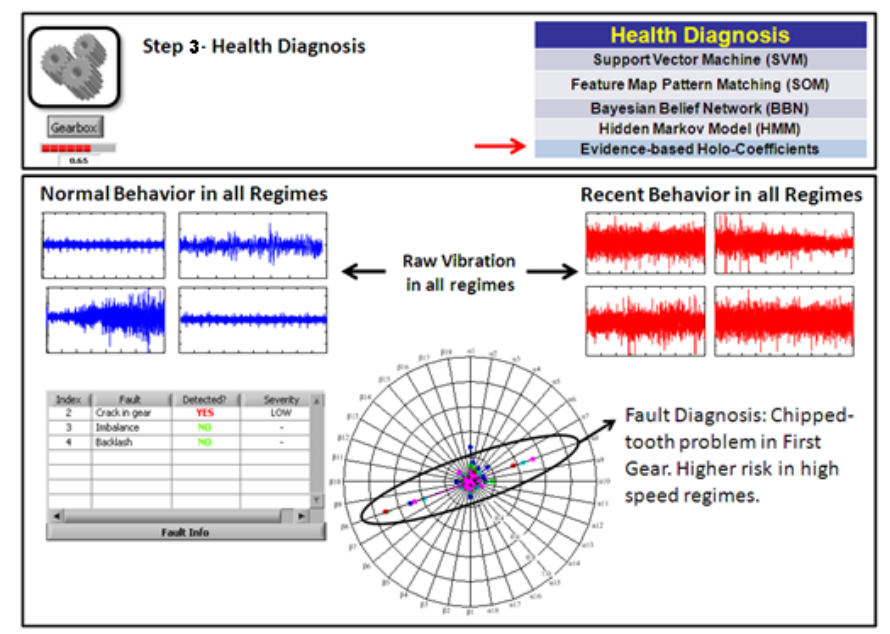

Fig. 13. Step 3 of wind turbine PHM: health diagnosis 

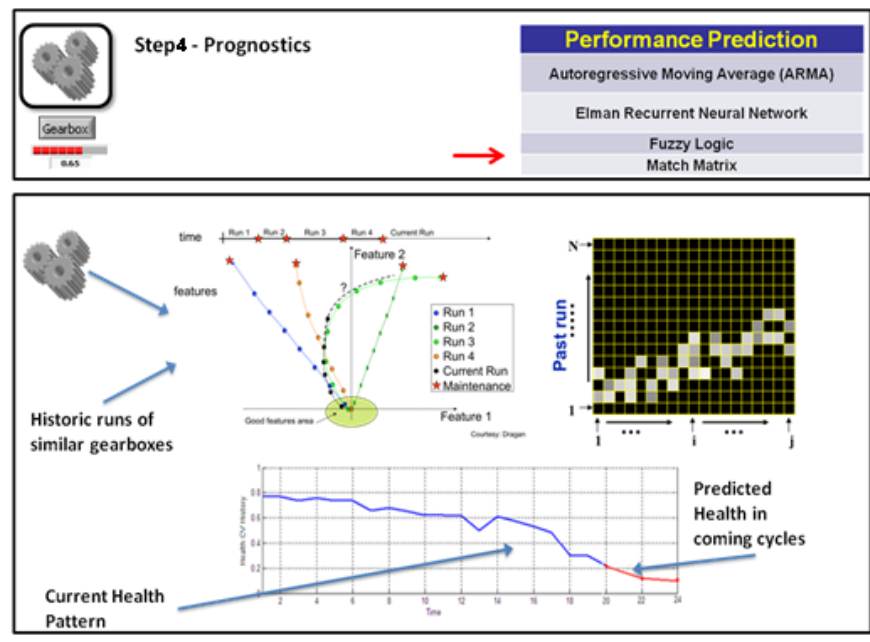

Fig. 14. Step 4 of wind turbine PHM: prognostics

\section{CONCLUSION}

The adaptive and multi-regime PHM system developed in this paper provides a more accurate health estimation, diagnosis and prognosis of critical components of wind turbines. The proposed research advances the understating of how to adaptively apply PHM to the various situations composed by dynamic operating conditions of wind turbines and the health condition of rotary components. This approach could be used in an intelligent and predictive maintenance program to minimize the time needed for inspection of components via on-line inspection, conducting remote site supervision and remote diagnosis, preventing catastrophic failures and secondary defects, preventing unnecessary replacement of components, allowing utility companies to be confident of power availability, improving designs and supporting further development of wind turbine. The reduction of maintenance risks and costs and improvement of reliability and efficiency will eventually make this green energy sector more competitive.

\section{ACKNOWLEDGMENT}

Great thanks should be given to research students and stuff in NSF IMS center, such as F.J. Wu, E.Lapira, H.Atat, L.X.Liao, T.Y.Wang, M.Abuali, D. Siegel, M. Ghaffari, and P.Johnson in National Instruments for their sharing and help in this research work.

\section{REFERENCES}

[1] U.S. Department of Energy report 20 Percent Wind Energy by 2030, "http://www1 .eere.energy.gov/windandhydro/pdfs/41869.pdf."

[2] American wind energy association (AWES),

[3] http://www.awea.org/publications/reports/AWEA-Annual-Wind-Report2009.pdf.

[4] C.C. Ciang, J.R. Lee and H.J. Bang, Structural health monitoring for a wind turbine system: a review of damage detection methods. Measurement Science and Technology 19 122001:20pp, 2008.

[5] H. Qiu, J. Lee, J. Ling, G. Yu, Robust Performance Degradation Assessment Method for Enhanced Rolling Element Bearings Prognostics, Journal of Advanced Engineering Informatics, Vol. 17, pp. 127-140, 2003.

[6] J. Lee, J. Ni, D. Djurdjanovic, H. Qiu, and H. Liao, Intelligent Prognostics Tools and E-Maintenance, Computers in Industry $57 \mathrm{pp}$. 476-489, 2006.

[7] L. Liao, and J. Lee, Design of a reconfigurable prognostics platform for machine tools, Expert Systems with Applications, 2009.

[8] GE Energy,

[9] http://www.gepower.com/businesses/ge_wind_energy/en/index.htm; http://www.gepower.com/prod_serv/products/oc/en/system_soft.htm.

[10] Vestas, http://www.vestas.com/en/wind-power-solutions/scada.aspx.

[11] Siemens, http://www.powergeneration.siemens.com/products-solutions services/products-packages/wind-turbines/.

[12] P. Tavner, J. Xiang and F. Spinato, Reliability analysis for wind turbines, Wind Energy, vol. 10, pp. 1-18, 2007.

[13] P. Tavner, G. V. Bussel and F. Spinato, Machine and converter reliabilities in wind turbines, PEMD 06, pp. 127-130, April 2006.

[14] H. Braam, and L. Rademakers, Models to analyse operation and maintenance aspects of offshore wind farms, ECN Report, 2004.

[15] W. Musial, S. Butterfield, B. McNiff, Improving Wind Turbine Gearbox Reliability. 2007 European Wind Energy Conference, Milan, Italy, May 7-10, 2007.

[16] Reinforcement learning,

[17] http://en.wikipedia.org/wiki/Reinforcement_learning

[18] T. Wang, J. Lee, The operating regime approach for prediction health prognostics, Proceedings of 62th Meeting of the MFPT Society: Failure Prevention for System Availability, pp. 87-98, 2008.

[19] J. Liu, D. Djurdjanovic, J. Ni, J. Casoetto and J. Lee, Similarity Based Method for Manufacturing Process Performance Predic tionand Diagnosis, Computers in Industry, vol. 58(6), pp. 558-566, August 2007.

[20] T. Wang, J. Yu, D. Siegel, J. Lee, A Similarity-Based Prognostics Approach for Remaining Useful Life Estimation of Engineered Systems, Proceedings of 2008 International Conference on PHM, pp. 1-6, 2008.

[21] PHM 2009 Data challenge Competition,

[22] http://www.phmsociety.org/competition/09. 